\title{
Metrology of low-photon light sources
}

\author{
Sergey Magnitskiy* \\ Faculty of physics and International Laser Center of Lomonosov Moscow State University, 119991 \\ Moscow, Russia
}

\begin{abstract}
In the report the parameterization of few-photon light sources is discussed. This question relates to a new area of research in quantum optics - the development of methods for characterizing optical components and devices used in optical quantum technologies that are supposed to be used in industry. Apart from, the recent results in this field obtained in the Laboratory of Experimental Quantum Optics and Nanophotonics of M.V.Lomonosov Moscow State University are briefly presented
\end{abstract}

At present, optical quantum technologies are emerging from the walls of laboratories and, as a result, issues of parameterization of quantum optics devices are become relevant. In 2016, the European Telecommunications Standards Institute (ETSI) issued the first preliminary standard [1] which established the rules for characterizing the optical components of quantum key distribution systems. Activity in this direction over the past 3 years has increased significantly. New specialized organizations and expert groups have emerged whose purpose is to develop preliminary standards for optical components and quantum optics devices. Obviously, the existing methods for characterizing classical light sources are insufficient to characterize quantum light sources. In [1] and in subsequent documents, the first procedures for characterizing pseudo-single-photon-sources are provided. But the proposed characterization methods are limited to measuring the Granger's $\alpha$-parameter and characterizing the quantum states of the generated light themselves remains open, although this is a key challenge for quantum optics [2].

Over the past 3 years, our laboratory has been developing methods for characterizing quantum optics devices. The work is carried out in 3 directions: 1) the development and characterization of quasi-single-photon SPR sources using quantum tomography, 2) the characterization of few-photon sources by the photon counting method, and 3) the development and characterization of photon counters using standardless methods. The schemes of a double-crystal SPR generators were developed which are not subject to the negative influence of the Migdall effect on the degree of entanglement of the states of generated photon pairs [3]. The method and the prototype of the device for certification of such sources has been proposed [4]. The effectiveness of certification was analyzed using various criteria of entanglement. The choice fell on the application of the parameter of proximity of quantum states - fidelity $(F)$. It was shown that when using such sources, $\mathrm{F}$ adequately characterizes the error $P_{\text {err }}$ of the distribution of the quantum cryptographic key,

\footnotetext{
* Corresponding author: sergeymagnitskiy@gmail.com
} 
which can be expressed through $\mathrm{F}$ as $\frac{1-F}{2} \leq P_{\text {err }} \leq 1-F$. The prototype of the device that we propose to use for certification is a quantum tomograph designed to measure the polarization state of photon SPR pairs according to the protocol proposed in [5]. Our implementation of such a device is described in [6].

Of undoubted interest is the characterization of the energy of few-photon light sources by the photon counting method, since the technical implementation of such a method with the use of modern multi-pixel photon counters is much simpler than using optical methods as Hong - Ou - Mandel or Brown - Twiss intensity interferometers, that is proposed to be used in [1]. In addition, measuring the statistics of photocounts makes it possible to determine all moments of the distribution, and not only the first two, as in the case of measuring the correlation function $g^{2}(0)$. In terms of equipment, we settled on multi-pixel SiPM photon counters. Despite significant advantages, this method has one significant drawback. When using it, it is not the photon statistics that are measured, but the statistics of the photocounts, which do not coincide in the case of finite quantum efficiency of the detector. Therefore, the problem of solving the inverse problem arises. Despite the simple analytical form of linking the photon statistics with the statistics of photocounts, the reconstruction algorithm is unstable relative to measurement error and, in practically interesting cases, does not allow the photon statistics to be correctly restored.

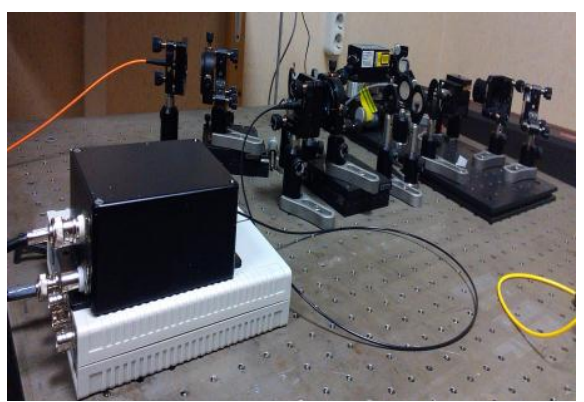

Fig. 1. General view of the single photon counters and time-digital convertors

We also briefly talk about the single photon counters and time-digital convertors that we developed (see Fig. 1), and discuss our proposed version of an improved Klyshko scheme of standardless measurement of the quantum efficiency of these devices. A distinctive feature of the developed counters is the ability to control and change all key parameters that affect the registration of single photons.

The more detailed presentation of the results discussed here can be found in our reports presented in the poster section of this conference.

In conclusion, I would like to express my gratitude to D.P. Frolovtsev, P.P. Gostev, D.P. Agapov, E.A. Mamonov, D.A. Yakovlev, A.S. Chirkin, A.A. Stifutkin (MEPhI) and A.V. Demin (VNIIOFI), who took and continue to take part in this work.

The work was partially supported by the RFBR (grant \#18-02-00849)

\section{References}

1. Standard: ETSI - GS QKD 011, ET Standards Institute (2016)

2. S.A. Magnitskii, D.N. Frolovtsev, D.P.Agapov, A.V. Demin, V.N. Krutikov, and G.G. Levin, Measurement Techniques, 60(3), 235 (2017)

3. D.N. Frolovtsev, A.V. Demin, V.N. Krutikov and S.A. Magnitskiy, unpublished results

4. D.N. Frolovtsev, S.A. Magnitskiy, Russian patent RU №2636808 (2016)

5. D.F.V. James et al. Physical Review A, 64(5), 052312 (2001)

6. S. Magnitskiy et al., Journal of Russian Laser Research, 36(6) 618 (2015) 